\title{
Acellular dermal matrix graft for ventral corporal lengthening orthoplasty in 2-stage proximal hypospadias repair
}

\author{
Shaofeng Wu^, Rong He, Jie Sun, Haiteng Zhao \\ Department of Urology, Shanghai Children's Medical Center, Shanghai Jiao Tong University School of Medicine, Shanghai, China \\ Contributions: (I) Conception and design: S Wu; (II) Administrative support: H Zhao; (III) Provision of study materials or patients: J Sun; \\ (IV) Collection and assembly of data: R He; (V) Data analysis and interpretation: R He; (VI) Manuscript writing: All authors; (VII) Final approval of \\ manuscript: All authors. \\ Correspondence to: Haiteng Zhao. Department of Urology, Shanghai Children's Medical Center, Shanghai Jiao Tong University School of Medicine, \\ Shanghai 200127, China. Email: zhaohaiteng@scmc.com.cn.
}

Background: Correcting ventral curvature (VC) by lengthening the ventral corpora using a graft has been verified feasible, but still has been associated with the recurrence. The use of acellular dermal matrix (ADM) in the setting of tissue reconstruction has captured the attention of many surgeons. There are few reports on the use of $\mathrm{ADM}$ exclusively as correction of VC. Thus, we evaluate the safety and effectiveness of repairing the defect with $\mathrm{ADM}$ for straightening the $\mathrm{VC}$ in proximal hypospadias repair.

Methods: We retrospectively analyzed the records of patients with proximal hypospadias who underwent ventral corporal lengthening with graft in staged repair from January 2013 to December 2019. Those with curvature greater than $30^{\circ}$ after urethral plate transection were enrolled. ADM was used for repairing the defect left by transversely transection of tunica albuginea. Patient outcomes were compared with the nonmatched control group who underwent the same procedure with tunica vaginalis (TV) repair. Patient demographics, operative techniques, complications, reoperations were summarized and compared between 2 groups.

Results: Forty-three patients underwent ventral lengthening with ADM repair after transverse urethral plate transection and 35 patients with TV patching respectively. At a mean follow-up of 10 months in those with the first-stage ventral lengthening, 5 of 43 (11.6\%) in ADM group was detected with recurrent VC, while 2 of 35 (5.7\%) in TV group were observed with recurrent curvature contemporarily ( $\mathrm{P}=0.363)$. At a mean follow-up of 46.8 and 45.3 months, persistent curvature in ADM group was not significantly different comparing to TV group (1/43, $2.3 \%$ vs. $1 / 35,2.9 \%$; $\mathrm{P}=0.883)$.

Conclusions: Ventral corporal lengthening using ADM graft may facilitate correction of VC without increasing the risk of urethroplasty complications. It offers a promising material that can be safe, effective and simple to use and provides psychological and aesthetic benefits. Additional series assessment and further randomized controlled trials will elucidate the clinical impact of using ADM with ventral lengthening.

Keywords: Proximal hypospadias; ventral curvature (VC); ventral corporal lengthening; acellular dermal matrix (ADM)

Submitted Aug 07, 2021. Accepted for publication Nov 10, 2021.

doi: $10.21037 / \mathrm{tp}-21-372$

View this article at: https://dx.doi.org/10.21037/tp-21-372

^ ORCID: 0000-0002-4668-5119. 


\section{Introduction}

Hypospadias is a common congenital anomaly of the phallus. For distal and mid shaft cases, the introduction of the surgical techniques during the last 40 years has evolved with great and reproducible outcomes-especially in terms of cosmetic results and functional success (1). On the other hand, proximal hypospadias is still a challenging surgical entity, even though significant variability in techniques have been recruited to improve the consequence during the last decades (2). For this subgroup, straightening the penis is an imperative step in hypospadias repair, several surgical techniques have been introduced to correct the curvature, including dorsal plication, urethral plate mobilization and ventral corporal lengthening with graft or flap $(3,4)$. Various grafts and biomaterials have been successfully used to correct the severe curvature, such as dermis (5), small intestinal submucosa (6), tunica vaginalis (TV) $(5,7)$. Previous studies have varied in different outcomes due to the graft contracture leading to the residual curvature (8). Human acellular dermal matrix (ADM), a decellularized cadaveric skin graft commonly used in the field of burn wound repair and tissue-engineered plastic reconstructive surgery. This material is prepared by multiple-step process to remove cellular components that might induce immune rejection. The safety and effectiveness of ADM implantation has been evaluated in plastic reconstruction (9). As a new type of tissue repair material, $\mathrm{ADM}$ has shown increasingly broad application prospects in treatment of urogenital diseases including penis, urethra and bladder. We, therefore, hypothetically presumed that the ADM graft could correct the ventral curvature (VC) by patching the ventral defect as that of TV flap provided.

The algorithm for repair was determined by the extent of VC after the urethral plate was transected. Patients with curvature 30 degrees or greater underwent 2-stage graft repair while those who with curvature less than 30 degrees were not included. The goal of this preliminary study is to evaluate many of the underlined factors that are ultimately related to achieving predictable and reproducible outcomes according to the materials used for ventral corporal lengthening. We present the following article in accordance with the STROBE reporting checklist (available at https:// dx.doi.org/10.21037/tp-21-372).

\section{Methods}

All patients who underwent hypospadias surgery had been retrospectively analyzed between January 2013 and December 2019. After receiving institutional review board approval, 43 consecutive patients with proximal defects in Shanghai Children's Medical Center who underwent ADM procedures were identified, ventral corporal curvature and the complications for straightening were determined. Patient outcomes were compared with those of 35 nonmatched control group with the same procedure in our hospital using TV flap. The main inclusion criteria were as follows: (I) primary patients with proximal hypospadias with severe curvature $\left(>30^{\circ}\right)$; (II) hypospadias repair by staged technique; (III) written informed consent from the parents about the allograft and the various methods of treatment to hypospadias. The main exclusion criteria were as follows: (I) presence of recurrent hypospadias, and/or disorder of sex development; (II) history of allergic or immune reaction to the allograft; (III) clinical abnormalities in routine laboratory examinations. The study was conducted in accordance with the Declaration of Helsinki (as revised in 2013). The study was approved by the ethics committee of Shanghai Children's Medical Center (SCMCIRB-W2021027). Informed consent was obtained from all parents or guardians. All the surgeries were performed by one of our authors (Zhao). Patient characteristics, preoperative evaluation, operative details, postoperative complications, residual VC and reoperations were obtained and compared between the two groups.

$\mathrm{ADM}$ was derived from skin of the donator and prepared by Beijing Qingyuan Biotechnology Company; it exhibited a porous scaffold by the process of removing the cellular components from the skin tissue. Acute/chronic toxicity, genetic toxicity, cytotoxicity, hemolytic toxicity, allergen detection, and degradability characteristics of the ADM were evaluated by the National Institute of Food and Drug Control, in accordance with the Chinese Criterion of Medical Device GB16886. The ADM material was sterilized by $12 \mathrm{kGy} \mathrm{Co}{ }^{60}$ irradiation.

Preoperative testosterone was used subjectively in accordance with the small appearance of the phallus and glans width before the first stage (10). Curvature was visually measured by the surgeon with the protractor. After completely degloving the phallus, the artificial erection was performed. In patients with curvature greater than 30 degrees, the urethral plate was transected, and the plate was dissected proximally and completely mobilized off from the corpora cavernosa. Artificial erection was repeated to demonstrate the VC. Residual curvature 30 degrees or greater was corrected by ventral corporotomy 

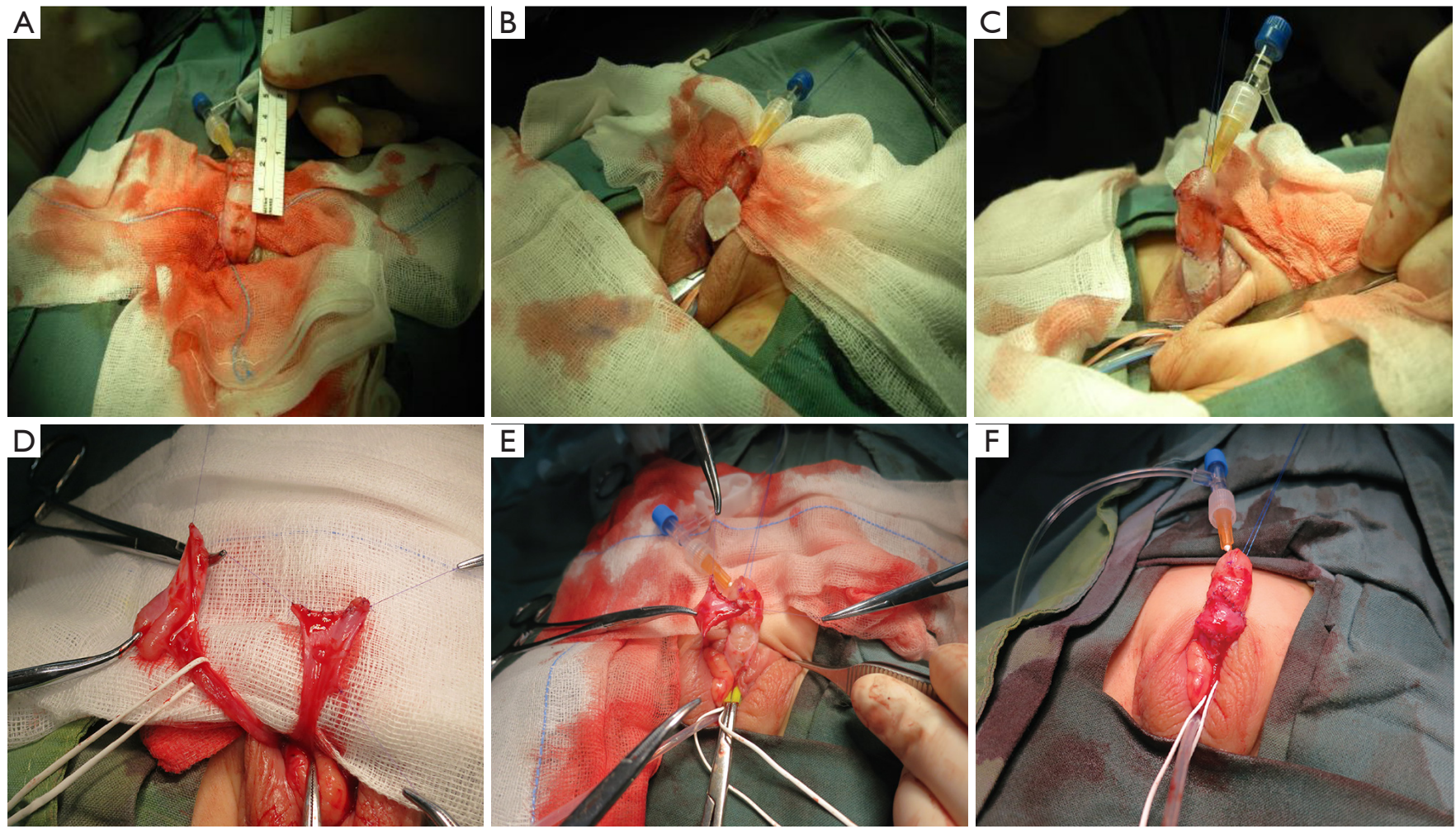

Figure 1 Main procedures of ventral corporeal lengthening using ADM or TV. (A) Transverse incision in the tunica albuginea at the point of curvature after artificial erection. (B) The ADM graft was placed to the defect and sutured to the tunica albuginea. (C) Artificial erection was repeated to ensure complete straightening the curvature. (D) The TV was dissected from the scrotum and (E) patched to the ventral aspect, care must be taken to prevent the torsion of the pedicle. (F) The TV flap was transposed to the ventral aspect of corporal defect. $\mathrm{ADM}$, acellular dermal matrix; TV, tunica vaginalis.

with graft or flap.

A transverse incision was implemented at the point of greatest curvature through the tunica albuginea with tourniquet compression, extending from 3 to 9 o'clock position. Careful dissections were carried out distally and proximally to expand the linear incision into ellipsoid defect that was later patched with an ADM graft (Figure 1A). To prevent the potential risk of recurrent curvature due to graft fibrosis or shrinkage of the materials, a little larger size graft had been harvested to repair the defect left by corporotomy without tension, normally $10 \%$ to $20 \%$ larger than the defect (Figure $1 B$ ). The graft was then anastomosed to the tunica albuginea to cover the underlying corporeal defect with 6-0 polydioxanone suture. Repeat artificial erection was achieved in all cases to make sure the successful correction and leakage assessment from the graft edge (Figure 1C). The control group was performed by the same surgeon with the similar techniques (2) as in dissection of TV (Figure 1D), transverse incision of tunica albuginea (Figure $1 E$ ), and lengthening the ventral corporeal aspect with TV (Figure 1F). We did not perform dorsal plication for any patients during the first stage operation. A suitable segment of transverse inner preputial flap was then harvested from dorsal aspect of the penis, the vascular pedicle must be protected and not to damage the viability of the flap, and then transposed to the ventral aspect of the shaft, making sure that the glans was incised in the middle to create the deep groove, allowing the flap to be sutured in place.

The second stage of the procedure was tubularization procedure. It was usually performed at a minimum interval of 6 months after the first operation. The diagnosis of recurrent $\mathrm{VC}$ was suspected by clinical examination and then confirmed by artificial erection during the second stage operation. The degree of $\mathrm{VC}$ found at the re-operation was determined by a protractor. If the curvature was $>30^{\circ}$, ventral lengthening was reoperated, if the $\mathrm{VC}$ was $<30^{\circ}$, dorsal plication was performed for those patients. This 
Table 1 Demographics and patient characteristics

\begin{tabular}{|c|c|c|c|}
\hline Baseline characteristics & ADM group & TV group & $P$ value \\
\hline Proximal penile & 15 & 11 & \\
\hline Penoscrotal & 19 & 20 & \\
\hline Perineal & 9 & 4 & \\
\hline Medial age at surgery, months & 14.5 & 16.2 & 0.586 \\
\hline Mean operative time, $\min$ & $120.7 \pm 21.7$ & $166.3 \pm 32.3$ & $<0.01^{*}$ \\
\hline Mean follow-up, months & $46.8 \pm 21.5$ & $45.3 \pm 22.3$ & 0.303 \\
\hline Pre-operative testosterone, $\mathrm{n}(\%)$ & $7(16.3)$ & $10(28.6)$ & 0.191 \\
\hline
\end{tabular}

*, $\mathrm{P}<0.05$ is statistically significant. ADM, acellular dermal matrix; TV, tunica vaginalis; IQR, interquartile range.

step also included tubularization of the neourethra over an 8 Fr silicon catheter and coverage with multiple layers of dartos flap or TV to reduce the risk of fistula formation. Glanuloplasty was performed concomitantly and correction of penoscrotal transposition was performed when necessary. All patients had the urethral catheter left indwelling for 14 days. Complications were reported by physical examination of the surgeons in clinic or by observation of children and parents during the postoperative follow-up.

\section{Statistical analysis}

The categorical variables were compared with chi-square analysis and Fisher's exact tests, and continuous ones with parametric or non-parametric tests, based on available data and distribution. Statistical analysis was done with SPSS, version 24.0. A P value of $<0.05$ was considered for statistical significance.

\section{Results}

From all the hypospadiac patients, we identified 78 patients who were operated by 2 -staged approach with $\mathrm{ADM}$ group 43 boys and those in TV group 35 counterparts; medial age at first stage was 14.5 (range, 8-75) months in patients with ADM group and 16.2 (range, 7-91) months in those with TV group. Of the 43 children who underwent ADM graft, 15, 19 and 9 had proximal penile, penoscrotal and perineal hypospadias, respectively. Of the 35 boys who underwent TV flap, 11, 20 and 4 had proximal penile, penoscrotal and perineal phallus defects, respectively (Table 1).
Preoperative testosterone stimulation was given in a similar proportion of children in ADM group and TV group (7 of 43 or $16.3 \%$ vs. 10 of 35 or $28.6 \%$ ). Mean operative time in ADM group $(120.7 \pm 21.7 \mathrm{~min})$ was significantly less than time spent in TV group $(166.3 \pm 32.3 \mathrm{~min})(\mathrm{P}<0.01)$. The medial curvature in ADM group was 40.5 (range, 30-80), [interquartile range (IQR), 35.5-60.3] degrees after transection of urethral plate, while the medial degree in TV group was 45.7 (range, 30-90), (IQR, 32.2-75.1) degrees $(\mathrm{P}=0.738)$ (Table 1).

Recurrent curvature was diagnosed at least 6 months after the first stage in 5 of the $43(11.6 \%)$ who underwent AMD straightening, while 2 of the 35 (5.7\%) who underwent TV lengthening, which was not significantly different $(\mathrm{P}=0.363)$. No other patients were examined and reported to have postoperative $\mathrm{VC}$ after first stage. Of those 5 patients, $\mathrm{VC}$ was measured by protractor to be 30 degrees or more in three patients $\left(30-45^{\circ}\right)$, recurrent VC was due to skin tethering and/or scarring of skin and dartos. Two additional patients were diagnosed with $\mathrm{VC}<30^{\circ}\left(15-20^{\circ}\right)$ from insufficient correction. Similarly, of those with recurrent VC in TV group, 2 were found at re-operation to have VC ranging from $15-30^{\circ}$ due to insufficient correction or corporal disproportion. Ventral lengthening was redone for those 3 patients with the curvature greater than $30^{\circ}$, while dorsal plication was performed for the other 4 patients less than $30^{\circ}$.

In our cohort, the median length of follow-up was $46.8 \pm 21.5$ (range, $12-72$ ) in ADM group vs. $45.3 \pm 22.3$ (range, 11-60) months in TV group. The overall surgical complications were observed in 15 patients $(34.9 \%)$ in 
Table 2 Complications of ventral corporeal lengthening

\begin{tabular}{lccc}
\hline Complications & ADM group & TV group & P value \\
\hline First stage bleeding/hematoma, (\%) & $2 / 43(4.7)$ & $7 / 35(20.0)$ & $0.035^{\star}$ \\
First stage infection, (\%) & $1 / 43(2.3)$ & $3 / 35(8.6)$ & 0.214 \\
First stage recurrent curvature, (\%) & $5 / 43(11.6)$ & $2 / 35(5.7)$ & 0.363 \\
Total complications, (\%) & $15 / 43(34.9)$ & $13 / 35(37.1)$ & 0.836 \\
Fistula, (\%) & $9 / 43(20.9)$ & $3 / 35(20.0)$ & 0.919 \\
Meatal stenosis, (\%) & $3 / 43(7.0)$ & $3 / 35(8.6)$ & 0.793 \\
Diverticulum, (\%) & $2 / 43(4.7)$ & $2 / 35(5.7)$ & 0.832 \\
Persistent curvature, (\%) & $1 / 43(2.3)$ & $1 / 35(2.9)$ & 0.883 \\
\hline
\end{tabular}

*, $\mathrm{P}<0.05$ is statistically significant. ADM, acellular dermal matrix; TV, tunica vaginalis.

GM group $v s$. that of 13 patients (37.4\%) in TV group with no different significance (Table 2). The most common complication was fistula, detected $9(20.9 \%)$ in ADM group vs. 7 (20\%) in TV group. The other complication required further disposal was meatal stenosis in ADM 3 (7.0\%) vs. TV 3 (8.6\%), and diverticula in ADM 2 (4.7\%) vs. TV 2 (5.7\%), respectively. During the limited follow-up, the persistent curvature in ADM group was not significantly different comparing to TV group $(1 / 43,2.3 \%$ vs. $1 / 35$, $2.9 \% ; \mathrm{P}=0.883)$. Corporal disproportion or contracted neourethra was found in these 2 patients with persistent VC in ADM group or TV group respectively, staged urethroplasty with the buccal mucosa had been performed for this patient. There was no urethral stricture in any patients after the second stage urethroplasty during the follow-up (Table 2).

\section{Discussion}

Correction of ventral corporal curvature is the principal step in hypospadias repair $(11,12)$. For most hypospadias cases, VC could be readily managed by de-gloving and dissecting the phallus along the Buck's fascia, however, despite the technique improvement, the management of proximal hypospadias remains one of the most challenging conundrums for pediatric urologists. Proximal hypospadias is concomitantly associated with curvature, especially when the penis size is small and short. The approach to correct severe curvature is still a controversial issue, and no maneuver is universally accepted (13). Dorsal plication, by shortening the dorsal aspect of corpora cavernosa, would be preferentially plausible in correction of curvature less than 30 degrees. Dr. Duckett advocated that dorsal plication was the most useful approach for straightening VC. However, this technique makes shortening an already short penis and, for a long run, seems irrationally to straighten severe curvature 30 degrees or greater in proximal hypospadias due to the higher incidence of recurrent bending and increased morbidity $(12,14)$. Another approach for penile curvature is to lengthen the ventral corpora by multiple incisions (fairy cuts) or by patching the tunica albuginea with natural tissue or other materials $(2,5,13,15)$. This maneuver has improved the curvature without compromising the penile length, which is especially beneficial to the hypoplastic, small sized penis. And also, as the corporeal disproportion pathologically involves on the ventral side, it sounds logical to intervene on the ventral aspect of the penis.

The TV has been proved to be an attractive alternative for correcting the VC due to sufficient vascular supply. Braga et al. (2) reported in 2007 the results of ventral lengthening using a TV flap to correct the curvature was favorable, with a $95 \%$ success rate. However, TV is often shortage due to previous orchiopexy or reservation for latter barrier flap to prevent the fistulae by covering the neo-urethra. Small intestinal submucosa grafts have been used for many years, but some authors (16) reported up to $20 \%$ complication of the patients with the fibrosis and recurrent ventral bending. ADM is manufactured by physical and chemical decellularized process to remove the antigenic components that might cause immune rejection. $\mathrm{ADM}$ is reportedly biodegradable and regenerative, while characterized with strong ability to induce angiogenesis in host tissue without causing significant inflammation, tissue fibrosis, or foreign body reaction. The extracellular matrix of three-dimensional cell scaffold structure with collagen fibers had been used for wound repair and plastic 
surgery, substantially extended in urological reconstruction in recent years, including bladder exstrophy (17), cloacal malformation (18), and urethral reconstruction. Upon implantation, this structure serves as a scaffold for cells to facilitate subsequent incorporation and revascularization. In our cohort, $\mathrm{ADM}$ is used mainly for patching the corporal defect left by ventral lengthening and reshaping the smallsized penis. It helps control the bleeding of the corpus spongiosum and prevent the formation of hematoma around the neo-urethral plate compared with the TV flap in the first stage repair (ADM $4.7 \% v s$. TV 20\%; $\mathrm{P}<0.05$ ) (Table 2). The orientation of the $\mathrm{ADM}$ is also important because most ADMs are usually polarized and have a dermal and basement membrane surface. The dermal surface should be in contact with the corpora cavernosa to enhance soft tissue incorporation and promote zones of adherence that can minimize the infection (ADM 2.3\% vs. TV 8.6\%; $\mathrm{P}=0.214)$. Traditional phallus reconstruction using autologous skin flaps has some defects, such as complexity of the techniques, shortage of the urogenital substitute, risk of necrosis of the skin flaps after surgery, and failure to achieve the satisfied cosmetic outcomes. The main disadvantage is the additional time requirement and the potential donor site morbidity from graft harvest, also confirmed by our results. Preparing the ADM graft and coverage of the corpora cavernosa could be completed without increasing the surgical time. In our series, the mean operative time in TV group was significantly longer compared to that of ADM group $(\mathrm{P}<0.01)$ (Table 1).

In addition, we compared the outcomes of phallus straightening between the ADM group and the pedicle flap group. It is reasonable to speculate that the additional vascular pedicle using the TV would allow for better straightening of VC compared with the free allograft coverage. However, our analysis revealed that the incidence of recurrent curvature was comparable between the two groups. In our series, 43 consistent patients involving the use of ADM, 5 (11.6\%) had recurrent curvature, while 2 patients $(5.7 \%)$ in $\mathrm{TV}$ had been observed recurrence after first stage orthoplasty, which was not significantly different $(\mathrm{P}=0.363$, Table 2). The observed curvature in the patients might have been complicated by urethral fibrosis and skin tethering, leading to inability of the urethra to grow, not merely to the insufficient correction at the ventral lengthening procedure. There is also a concern about the incidence of curvature recurrence of ADM graft possibly due to lack of growth. ADM generates physiological cues that mimic the native tissue microenvironment that could promotes cell proliferation and facilitate subsequent cell migration and differentiation. For the patients in our study, thin sheets of ADM in the range of $1-1.5 \mathrm{~mm}$ in thickness are preferred as this may facilitate more rapid incorporation, adherence, and revascularization. Furthermore, we confirmed that late onset of persistent curvature has only been detected in ADM group for 1 patient (2.3\%) as well as TV flap in 1 of 35 patient (2.9\%) with limited follow-up without significant difference ( $\mathrm{P}=0.883$, Table 2). But longterm follow-up, especially after the puberty, still need to address these issues.

Although we did not observe severe fibrosis formation, graft contract or severe recurrent curvature at the ventral defect site in this study, its potential risk could not be neglected. In order to avoid this potential risk, we preferred to choose a little larger graft (up to $20 \%$ larger than the defect area) to repair the defect left by ventral corporotomy. Actually, 3 patients with $>30^{\circ} \mathrm{VC}$ after the first stage in $\mathrm{ADM}$ group undergoing re-operation mainly were due to the improper disposal of the graft during initial period. Thereafter the anastomotic site was covered by the pedicled island flap transposed from dorsal aspect, which may promptly provide the nutrients and better vascular supply on the surface of the substitution, especially on the devascularized matrix surface. With appropriate tailoring of the graft and meticulous techniques, the risks could be minimized to ensure adequate coverage of the graft with well-vascularized tissue layers.

To date, there is no published research of the ADM as a graft for ventral corporal lengthening. In this study, we confirm that ADM graft to lengthen the ventral aspect of tunica albuginea is effective to all the patients with severe VC greater than 30 degrees in proximal hypospadias in more than 3 years follow-up. The off-shelf availability, less operative time and lack of donor site morbidity of ADM graft made it a superior alternative for correcting the severe curvature in proximal hypospadias. Application of the graft to the tunica albuginea of the corpora cavernosa provides a more solid base to allow better fixation of the graft, reducing the bleeding from the anastomosis, and decreasing contracture during healing afterwards. Also, theoretically there should be much more well shape of the penis after ventral lengthening other than dorsal plication, hence, a greater cosmetic appearance, and less psychological affect.

Limitations of our research include its non-randomized, retrospective study at a single institution and the fact that the assessment of the curvature by a single surgeon. The preoperative assessment is, like other similar research, all 
subjectively performed including the quality of urethral plate, degree of VC and quality of spongiosum. And also, we lack standardized measures of curvature, particularly early in this inclusion period. Limited follow-up into puberty, when the increased phallus growth may deteriorate the degree of curvature, restricts us to find the difference. Despite these limitations, it is believed that lengthening the ventral aspect by ADM is a feasible approach in cases of severe curvature with proximal hypospadias (19). Clearly, a larger series with long term follow-up is imperative to assess utterly the outcome of proximal hypospadias surgery.

\section{Conclusions}

From current outcome, ventral corporal lengthening using ADM may facilitate correction of $\mathrm{VC}$ in proximal hypospadias without increasing the complications. ADM offers a promising material that can be safe, effective and simple to use without tissue harvesting and donor site damage. It may be an effective alternative for correction of $\mathrm{VC}$ in proximal hypospadias repair with psychological and aesthetic benefits.

\section{Acknowledgments}

Funding: This work was supported by the National Science Foundation for Young Scientists of China (grant No. 81100477 to SW); and Shanghai Science Foundation (grant No. 11ZR1423300 to SW).

\section{Footnote}

Reporting Checklist: The authors have completed the STROBE reporting checklist. Available at https://dx.doi. org/10.21037/tp-21-372

Data Sharing Statement: Available at https://dx.doi. org/10.21037/tp-21-372

Peer Review File: Available at https://dx.doi.org/10.21037/ tp-21-372

Conflicts of Interest: All authors have completed the ICMJE uniform disclosure form (available at https://dx.doi. org/10.21037/tp-21-372). The authors have no conflicts of interest to declare.

Ethical Statement: The authors are accountable for all aspects of the work in ensuring that questions related to the accuracy or integrity of any part of the work are appropriately investigated and resolved. The study was conducted in accordance with the Declaration of Helsinki (as revised in 2013). The study was approved by the ethics committee of Shanghai Children's Medical Center (SCMCIRB-W2021027). Informed consent was obtained from all parents or guardians.

Open Access Statement: This is an Open Access article distributed in accordance with the Creative Commons Attribution-NonCommercial-NoDerivs 4.0 International License (CC BY-NC-ND 4.0), which permits the noncommercial replication and distribution of the article with the strict proviso that no changes or edits are made and the original work is properly cited (including links to both the formal publication through the relevant DOI and the license). See: https://creativecommons.org/licenses/by-nc-nd/4.0/.

\section{References}

1. Long CJ, Canning DA. Hypospadias: Are we as good as we think when we correct proximal hypospadias? J Pediatr Urol 2016;12:196.e1-5.

2. Braga LH, Pippi Salle JL, Dave S, et al. Outcome analysis of severe chordee correction using tunica vaginalis as a flap in boys with proximal hypospadias. J Urol 2007;178:1693-7; discussion 1697.

3. Shukla AK, Singh AP, Sharma P, et al. Two Stages Repair of Proximal Hypospadias: Review of 700 Cases. J Indian Assoc Pediatr Surg 2017;22:158-62.

4. Braga LH, Lorenzo AJ, Bägli DJ, et al. Ventral penile lengthening versus dorsal plication for severe ventral curvature in children with proximal hypospadias. J Urol 2008;180:1743-7; discussion 1747-8.

5. Leslie JA, Cain MP, Kaefer M, et al. Corporeal grafting for severe hypospadias: a single institution experience with 3 techniques. J Urol 2008;180:1749-52; discussion 1752.

6. Kropp BP, Cheng EY, Pope JC 4th, et al. Use of small intestinal submucosa for corporal body grafting in cases of severe penile curvature. J Urol 2002;168:1742-5; discussion 1745.

7. Hayashi Y, Kojima Y, Mizuno K, et al. Demonstration of postoperative effectiveness in ventral lengthening using a tunica vaginalis flap for severe penile curvature with hypospadias. Urology 2010;76:101-6.

8. Harper L, Michel JL, Sauvat F. Preliminary experience using a tunica vaginalis flap as the dorsal component of 
Bracka's urethroplasty. BJU Int 2017;119:470-3.

9. Sobti N, Ji E, Brown RL, et al. Evaluation of Acellular Dermal Matrix Efficacy in Prosthesis-Based Breast Reconstruction. Plast Reconstr Surg 2018;141:541-9.

10. Rynja SP, de Jong TPVM, Bosch JLHR, et al. Testosterone prior to hypospadias repair: Postoperative complication rates and long-term cosmetic results, penile length and body height. J Pediatr Urol 2018;14:31.e1-8.

11. Snodgrass $W$, Bush N. Surgery for Primary Proximal Hypospadias with Ventral Curvature $>30^{\circ}$. Curr Urol Rep 2015;16:69.

12. Snodgrass W, Bush N. Staged Tubularized Autograft Repair for Primary Proximal Hypospadias with 30-Degree or Greater Ventral Curvature. J Urol 2017;198:680-6.

13. Schlomer BJ. Correction of Residual Ventral Penile Curvature After Division of the Urethral Plate in the First Stage of a 2-Stage Proximal Hypospadias Repair. Curr Urol Rep 2017;18:13.

Cite this article as: $\mathrm{Wu} \mathrm{S}, \mathrm{He} \mathrm{R}$, Sun J, Zhao H. Acellular dermal matrix graft for ventral corporal lengthening orthoplasty in 2-stage proximal hypospadias repair. Transl Pediatr 2021;10(12):3151-3158. doi: 10.21037/tp-21-372
14. Snodgrass W'T, Bush NC. Management of Urethral Strictures After Hypospadias Repair. Urol Clin North Am 2017;44:105-11.

15. Ritchey ML, Ribbeck M. Successful use of tunica vaginalis grafts for treatment of severe penile chordee in children. J Urol 2003;170:1574-6; discussion 1576.

16. Soergel TM, Cain MP, Kaefer M, et al. Complications of small intestinal submucosa for corporal body grafting for proximal hypospadias. J Urol 2003;170:1577-8; 1578-9.

17. Bonitz RP, Hanna MK. Use of human acellular dermal matrix during classic bladder exstrophy repair. J Pediatr Urol 2016;12:114.e1-5.

18. Ladd AP, Rescorla FJ, Eppley BL. Novel use of acellular dermal matrix in the formation of a bioprosthetic silo for giant omphalocele coverage. J Pediatr Surg 2004;39:1291-3.

19. Snodgrass $W$, Bush NC. Persistent or recurrent ventral curvature after failed proximal hypospadias repair. J Pediatr Urol 2019;15:344.e1-6. 